\title{
BIJDRAGE TOT DE KENNIS DER DIALECTEN OP HET EILAND TIMOR.
}

DOOR

\author{
DR. J. G. F, R I EDEL.
}

Voor zoover ik dit door vergelijking heb kumnen vaststellen, worden er op het eiland Timor drie hoofddialecten, met name Timol, Belu en Helo in de landschappen Dawan, Tetuun en Marae gebezigd, welke echter door de oudsten, die mij inlichtingen verstrekten, alweder in tal van onderdialecten verdeeld zijn. Van deze laatste, vooreerst meer in het bijzonder van die van Tapenu, of Taibeno, Sonabai-ana, Oëmatan of Amakono en Djenilo, geef ik hier eene proeve, bestaande uit veertig volzinnen in het Taibenosch, een verdicht verhaal in het Sonabaisch, eene korte beschrijving van den aanleg eener djagongveld en van de verrichtingen bij tooverij wegens ziekte, in het Oëmatansch, zoomede eenige strophen van Djenilosche liederen, met name Samahare, die bij alle feesten en bij den padioogstgezongen worden. Lakumerin - treurliederen bij sterfgevallen en bewaking der lijken door vrouwen aangeheven, Tebevunu - oorlogsliederen waarin de vijand beleedigd wordt, Daisowan — schimpliederen door mannen en vrouwen bij feestelijke gelegenheden aangeheven en Loir - zangen door jonge lieden in den maneschijn gezongen. Het Djenilosch behoort tot het Belusch dialect, terwijl het Taibenosch, Sonabaisch en Oëmatansch onder Timol gerekend worden. Bij den oorspronkelijken tekst heb ik eene Nederlandsche vertaling en toelichtingen gevoegd.

In het Timolsch en Helosch dialect heeten de volkszangen Wakosala - vreugdeliederen bij feestvieringen en verheerlijking van aanzienlijken, van helden en overleden krijgers, ook gedurende den padioogst gebezigd; Olotloo, Samahare, Hunulau of Daisowan, en Tebediak - liederen bij het bestijgen van nieuwe woningen en bij gewone feesten door mannen en vrouwen die elkander op bedekte 
wijze beschimpen, aangeheven; Wonehunu en Boneleke - krijgsliederen die in gewone tijden niet mogen worden gebezigd, door mannen bij het binnenbrengen in de negari van de door hen gesnelde koppen gezongen; Hanonolalan, Makarereu, of Lakumerin - treurzangen bij lijken aangeheven en Loir, of Hering - liederen die door jonge lieden bij verschillende gelegenheden op velerlei wijzen worden gezongen.

\section{Tatbenosch dialect.}

Homoko me neem, 1 van waar komt gij? Au oko Lotes neem, ik kom van Rote.

Heu nao on mee, werwaarts wilt gij thans gaan?

Nao pa Kopan, ik wil naar Kupang gaan.

Soba sosa ane, wil mijn vriend rijst koopen?

Pikol mese lopeo vauk, hoeveel gulden kost een pikol?

Pikol mese lopeo haa, een pikol kost vier gulden.

Upan matane, dit is te duur.

$V e e$ oël kaleo, geef mij een weinig drinkwater.

Mui sabota au soos kaleo, hebt gij tabak, ik wil een weinig koopen.

Kuan $i$ kanaan saa, hoe heet deze negari?

Nakaav kanaan seko, hoe heet het (besturend) hoofd?

Nenoia an nao, ik wil thans terugkeeren.

Kesam tokoloo, blijf niet lang zitten.

Manas seen tamen, de zon gaat onder.

Mabiia naula naek, het zal heden nacht zwaar regenen.

An toti kisna ani naek, ik hoop dat er geen zware wind komt.

Au ume loli naek kaa, mijn huis lekt op veel plaatsen.

Au lele kavei seen, mijn veld is nog niet beplant geworden.

Soba lom hemuan saan, wat wil (mijn) vriend eten?

Au bikase, (dit is) mijn paard.

Bidjael nasosa bime, waar hebt gij dien buffel gekocht?

Nenoian ika naek, wij hebben heden veel visch.

Lekae masa biin, wanneer is het hier markt?

$A u$ soba atoni namatee, mijn vriend is vermoord geworden.

Leka es namatee, wanneer is hij vermoord geworden?

Autau san saa, wat heeft hij misdreven?

1 Voor de lange a, e, i, o en u gebruik ik aa, ee, ii, oo en uu. De u wordt sls oe in het Nederlandseh uitgesproken. 
Natus bale baen bale, natus naa baen naa, goederen behoort men met goederen, bloed met bloed te betalen.

Auvee naou, waar wil hij gaan?

Naka namena naek, mijn hoofd doet veel pijn, ik heb hoofdpijn.

Au vee noka noka biulan tenai, gisteren ben ik den geheelen dag tot aan den avond in den regen gebleven.

Pene pikul mese upan lopeo mese, een pikol djagong kost een gulden. Manu haa upan lopeo mese, vier kippen kosten een gulden.

Noa voa mese loit nua, een kalapanoot kost twee centen.

Atoni Lote kan naan see, hoe heet die Rotenees?

Ainaav nok amaav kan naav seko, hoe heeten zijne ouders?

Au um nahe, ik heb honger.

Uheem miin oёl noa, wilt gij kalapawater drinken?

Au naben teik nameen, ik gevoel pijn in de buik.

Muhaan ö̈l maputu, kook een weinig warm water.

A a nteekeningen. Ho, gij. Mo, van. Neem, nema, komen. $A u$, ik. Mao, gaan. Sos, soos, koopen. Ane, rijst, padi. Vauk, hoeveel. Upan, prijs. Matane, duur. Vee, geven. Ö̈l, ö̈, water. Kaleo, een weinig. Sabot, tabak. Kuan, negari. Kanaan, naan, naav, naam. Saa, wat. Nenoia, heden, thans. Naka, hoofd ook chef. Toko, zitten. Loo, lang. Manas, zon. Naula, regen. Anin, wind. Ume, huis. Loti, lekken. Lele, veld. Seen, sene, planten, beplanten. Kave, nog niet. Hemua, mua, eten. Saan, saa, wat. Bikase, paard. Bidjael, buffel. Nasosa, vid. sos. Ika, visch. Masa, pasar. Atoni, mensch, iemand. Namatee, gedood, mate, mupau, dooden. San, sana, schuld, misdrijf. Natus, utus, schuld. Bale, goederen. Baen, betalen. $N a$, naa, bloed. Namena, namen, ziek zijn, pijn voelen. Pene, djagong. Mese, mees, een. Haa, vier. Noa, kalapanoot. Manu, kip. Nua, twee. Ainaav, ina, moeder. Amaav, ama, vader. Nahe, namna, na, honger. Miin, ïn, drinken. Teik, buik. Muhaan, uhan, kooken. Maputu, putu, warm, heet.

\section{SonabaI-ANaSCH DIALECT.}

$\mathrm{Bi}$ mĕnasi aton monlees in umle nanan etne ivo nanvau. Esnaa atoni naev nanmoë nanmeku iiv gěwes, neki klit hau lakoëv oke antao sismeto seka bi nanan moë vuli nok ivo-ivonan. Nok mawe nanne nemmee ivo ikonua ikov ees namnasi nok iko munvees. Nuasin naon nateev mekunan ok at naskok sisivon. Ivo amnasi tan kan pilsaiv meku nan, oke in namna in naen naksut non. Maa le iiv munivee 
kan oma kanaka lolou, natuin in palun ne kun, laba akanama tam neu meku nanan. Nan in neku nalale namseen in hen poin van, ma oneen kan teniveen, oko miu mansenpee le naev nan na mat iiv muniv nan.

Vertaling. In de woning van een oud man waren vele ratten. Uitdienhoofde makte hij een rattenval, deed daarin hars van den lakoëv (Artocarpus incisa) en een stuk gedroogd vleesch dat gebraden werd als lokaas voor de vele ratten. In denzelfden nacht kwamen er twee ratten, een oude en een jongen. Beide liepen om de val en roken den geur van het gebraden vleesch. De oude rat vertrouwde de val niet en, ofschoon hongerig, verwijderde hij zich met spoed. Maar de jonge rat overwoog niet lang, volgde slechts zijn lust en kroop in de val. Toen hij verzadigd was, wilde hij weder terugkeeren, doch konde niet en den volgenden dag werd de jonge rat door den ouden man gedood.

Aanteekeningen. Mrnasi, nasi, naas, oud. Aton, mensch, iemand. Monlees, mees, ees, es, een. Umle, woning, huis. Etne, eet, zijn, aanwezig zijn. Ivo, ratten, muizen. Nanvau, veel. Esnaa, alzoo. Mö̈, mo, maken, doen. Nan meku, val, vanger, heke, heek, vangen. Iiv vid ivo. Gěwes, een. Klit, gom , hars, kleefsel. Hao, boom. Antao, leggen, plaatsen. Sismeto, gedroogd vleesch, sisi, vleesch, meto, droog. Seka, gebraden. Vuli, lokaas. Mawe, nacht. Nanne, dezelfde. Nemmee vid nema, komen. Iko, staart. Ikov ees, een staart. Munvees, jong, pas begonnen. Nuasin, beide. Nateev, rondom. Aat, ruiken. Naskok, navoo, geur. Sis ivon, vleesch voor de ratten bestemd. Kan, kal, kaleo, weinig, minder. Pilsaiv, vertrouwen, gelooven. Naen, main, wegloopen. Naksut, zich met spoed verwijderen. Oma, munau, overwegen. Lolou, lang. Natuin, mutuin, volgen, muniv, achter. Kun, verlangen, wensch. Akanama, kruipen. Namseen, zat gegeten. Poin, poinone, naar buiten (gaan). Teniveen, veeten, meer. Oko, ok, en. Miu, den volgenden dag. Mansenpee, morgen, ochtend. Mat, dooden.

\section{Ö̈matansch DIalect.}

He moë lale pena a oët al hau meshe sin naklati lekleok am ot miaka. Noka lel es mis naas wis kon hen meto. Met wen motoë al hau nan meki ai hen putu. Ka putuv oke natuk he sona. Oke naeta bahan leka wahian nateem lele. Nan pao ulan nem sen pena nok woko, 
nok vuel, nok laku, nok loli, nok al wian-wian, nok ine kan. Nawa an moni meshen tov na sona. Leka pena nasuv lele aton angkiso pena nan kapu neev in nao mami mĕnane. Niu nit mĕnane in naton mĕnane, "au pena an leu". Oke mĕnane natoti, "pena nan in leu onme", in natae, "pena nan in leu ka nesav". Mĕnane naton le lele aton, "ho heemnao mami manu nua nai me es, manu es op metan. He manu an metan ho vua avu. Muton ho ka sanav sa ho mes heem nao mami malo he hainiki lele nan. He malo sa saa sa nahe malo moi niki naik malo maputu, heem hainiki.lele nan, pisil meki oël he pena nan es." $\mathrm{Na}$ muni neno woës lele aton nao nono lele pena an, na leok wen leka. Pena an meto oke lele aton il oke antao pune woës niu aistes neki noi ume.

Vertaling. Wil men een djagongveld aanleggen, dan moet men de boomen omkappen en het hout goed in stukken hakken. Heeft men een stuk grond van voldoende uitgestrektheid ontgonnen, dan laat men het hout (boomstammen en takken) droog worden. Droog zijnde steekt men het een en ander met vuur totdat alles afbrandt. Als dit niet geschiedt, dan brengt men datgene dat niet afgebrand is op zijde bijeen. Alsdan begint men een heg te maken totdat het geheele veld omheind is. Vervolgens wacht men op regen om djagong, pompoenen, katjang, ubi, patatas en andere gewassen met een opgeruimd hart te planten. Als het gras opgroeit, moet het veid gewied worden. Wanneer de djagong gebloeid heeft en de eigenaar bemerkt dat de vruchtzetting niet behoorlijk geschiedt, dan moet hij een toovenaar zoeken. Dezen gevonden hebbende, moet hij zeggen : "mijn djagong wordt niet goed". De toovenaar vraagt: "wat scheelt er aan", waarop de eigenaar antwoordt: "de djagong wordt niet goed, want er komen geene korrels." Alsdan zegt de toovenaar aan den eigenaar van het veld: "wanneer dit zoo is, moet gij zoeken twee hoenders, een rooden haan en een zwarte kip. De zwarte kip offert gij aan de aarde. Geeft daarbij te kennen dat gij niet schuldig zijt en zoekt eenige kruiden op om het veld koud te maken. Welke kruiden die ook zijn mogen, wanneer deze slechts verkoelend werken en geene verhittende kruiden zijn om het veld koel te maken, mengt de kruiden met water en begiet daarmede uw veld opdat de djagong korrels geve ". Tien dagen daarna bezoekt de eigenaar het veld en ziet dat alles goed is geworden. Is de djagong droog, dan plukt de eigenaar de klossen; bindt ze tien aan tien bijeen in bossen en neemt ze mede naar zijne woning. 
A anteekeningen. Lale, lele, lel, veld, tuin, akker. Oèt, ote omkappen, slachten. Meshe, Taohe, he, moeten. Naklate, fijn, in stukken hakken. Lekleok, leko, goed. Aka, stukken hout. Misnaas, liggen laten. Meto, met, droog. Motoe, branden. Ai, vuur. Henputu, kaputuv, geheel verbranden. Natuk, nako, vegen. Naeta, beginnen. Bahan, wahan, heg. Nanpao, pao, wachten. Sen, sene, planten. Woko, pompoen. Laku, ubi. Vuel, katjang. Loli, patatas. Wianwian, alles. Nawa, onkruid. Moni moli, leven, groeijen. Iov, wieden. Nasuv, bloeien. Angkiso, kiso, zien. Mami, zoeken. Mènane, toovenaar. Leu, naleu, bedorven, niet goed. Natae, ta, antwoorden. Onme, hoe. $M e$, rood. Metan, zwart. Vua, offeren. Avu, aarde, grond. Muton, zeggen. Sanav, kav, niet. Malo, geheime middelen, kruiden. Haniki, koud, koel maken. Sanaho, zij het ook. Pisil, umis, met water kneden. Muni, achter, daarna. Neno, dag, Wö̈s, tien. Leok, zien, bevinden. Leka, leko, goed. Antao, tao, maken, doen. Pune, klossen. Aistes, een bos. Neki, meki, brengen.

Etan atoni es namen nak taina sinan nao toti totkit atoni eshe hinahu nehe auni. Nael nehe auni naton nak Us neno namaut anmoë menas nan, lo toti vua Us neno nok widjael keso me, henael he menas hen leoko. He kisatoni ote bidjael hen mate henaan bidjael in luken leu, na nael djael in luken nok mes, hen haak hen atoti hen ton Us neno, us anmoë menas nael au he vua he Us neno nok iv djael keso me, atoni nael name nak taina na leokwen. Le lo neno hitu kan hen leohe kisatoni tote le atoni nael nahu nehe auni kison ten nui le menasnae. Nael nehe auni naton toti henvua ho nitu hoaina nok hoama nok es vavi me. Le atoni amenas ka topu lo in lelu nan manu es ane ve atoni nae le ahine nehe auni kison tebi manu uwan. Atoni nan nahin nehe auni naki u manu tait naton toti henvua homsaa ho aina nok ama in sĕmanan nok vavi me es. Oke henvua sina lolo vavi es an kis vavi aten atoni in nannamel lov naleoko nan neno nim atoni amen atnan na leoko vi.

Vertaling. Heeft iemand eene buikziekte, dan moet men bij den toovenaar gaan om hem te raadplegen. Zegt hij dat de heer Zon de ziekte veroorzaakt heeft, dan behoort men te offeren aan den heer Zon een rooden buffel van het mannelijk geslacht, opdat de zieke moge herstellen. Alsdan wordt een buffel geslacht en staande met een stuk 
van het rechteroor en eenig rijst in de hand smeekt men aan den heer Zon, de heer die den persoon ziek gemaakt heeft en aan wien de roode buffel van het mannelijk geslacht geofferd wordt, om den zieke te doen herstellen. Wanneer na zeven dagen de zieke niet geneest, dan vragen de lieden weder aan den toovenaar om te zien hoe of het met den zieke gesteld is. De toovenaar zegt dat er een rood varken aan de geesten van den vader en de moeder moet worden geofferd. Is de zieke daarmede niet tevreden, dan laat hij een kuiken aan den toovenaaar ter hand stellen om den ader te raadplegen. De toovenaar dit gedaan hebbende, zegt dat men aan de geesten der ouders nog een rood varken moet offeren. Alzoo slachten zij een varken en onderzoeken de lever of de zieke nog genezen zal. Vijf dagen daarna is de zieke gewoonlijk geheel hersteld.

A a nteekeningen. Etan vid eet. Nak taina, nak, bloed, tai, faeces, bloed in zijn faeces. Sinan, si, zij. Toti, verzoeken. Eshe vid es. Hinahu, nahu, ahu, weten, er bedreven in zijn. Nehe, he, vadem, nehe auni, de spiets vademen, gewone benaming voor toovenaars die met het vademen van een piek hun proces beginnen. Naton, muton, ton, zeggen. Us, usi, heer. Neno, dag, zon. Mènas, ziekte, warm zijn. Widjael, bidjael, djael, buffel. Keso, het mamnelijk geslacht van dieren. $M e$, rood. Henael, opdat. Leoko, leko, goed, gezond, welvarend. Henaan, aan, nemen. Luken, oor. Leu, regts. Mes, ménes, ontbolsterde rijst. $V u a$, offeren. $I$, iv, deze. Leokwen vid leoko. Leohe vid leko. Kisatoni, zij lieden. Kison vid kiso, zien. Henvua vid vua. Nitu, geesten voorvaderen. Hoaina vid ina, moeder. Hoama vid ama, vader. Vavi, varken. Topu, ontvangen, willen, aanvaarden. Lelu, lelo, zenden, laten doen of verrichten. Vee, ve, geven. Uwan, ingewand, eigenlijk ader bij den testiculi waaruit voorspeld wordt. Tait, ingewand, vid teik. Lolo, slachten van vee. Kis, onderzoeken, raadplegen. Aten, lever. Nannamel, vid namen. Lov, loov, wachten. $V i$, geheel, door en door.

\section{DJENILOSCH DIALECT.}

1. Wehali we moometan, matetoëk nai naik, Diruma talin, Wehali talin, Diruma talin.

Samahare lied.

2. Kuda kaku laledik tama Naemuti, Toësinola leut rohan tama Naemuti. 
Varansisuk mai nodi kedan daun ida

Bua humur ida tuli daun ida.

Lakumerin lied.

3. Lari akadirun neon kaikona

Neon lalo lalokan neon kaikona.

Kulan uma mane saimai

Sai mai lesu dato sai mai.

Tebevunu lied.

4. Beli odomatan beli momoon

Beli momoon keti sedilia ruma.

Daisowan lied.

5. Kiakami nuudaar vatu soë lerek

$\mathrm{Nu}$ u vatu soë lerek voti soë soruk.

Manu ida kokorek nalu koi tadu

Koi tadu sadia ina no aman.

Loir lied.

Vertaling. 1. Het water van Wehali is helder, spreekt toch langzaam (en duidelijk) over de teekenen (het gebeurde) te Diruma of Dirmaa en Wehali.

(Aansporing om iets duidelijk en zonder zich te haasten mede te deelen.)

2. Het paard, dat zijn touw heeft losgebroken, is gegaan naar Naemuti, volgt de voetsporen, de stappen gaande naar Naemuti.

Varansisuk is gekomen, medebrengende een naald, een handvol pinangnoten in waarde gelijkstaande aan een naald.

(Verklaring dat men den doode niet volgen kan en dat een der bloedverwanten een onbeduidend geschenk voor den doode heeft medegebracht.)

3. De vijanden gelijken in aantal op de bladen der tuaboomen, dit doet goed aan het hart dat aan velerlei denkt.

De zakdoeken (maagden) van de familie der vrouw komen daar, zij komen als gevolg (zakdoeken) van den vorst.

(Mededeeling dat het goed is voor afleiding om vele vijanden te zien en dat de maagden, den koppensnellers ter eere naar buiten komen.)

4. Zingt voor de poort van den vorst, zingt op behoorlijke wijze, doch overtreedt zijne bevelen niet. 
(Randgeving on waneer men de vorten vleien wil, wogrziclitig te zijn.)

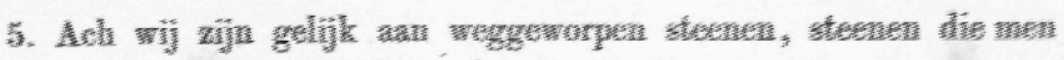
opneent on nogmals veruler te werper.

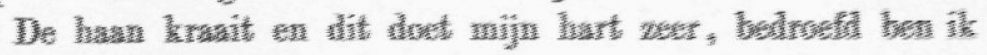
denkendo an mijue onders.

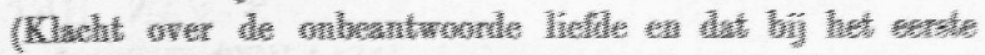

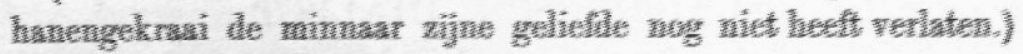

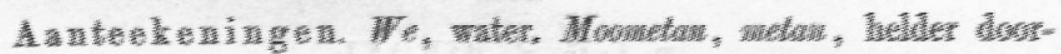

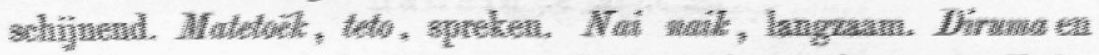

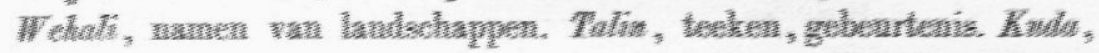

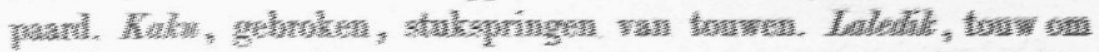

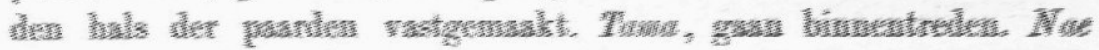

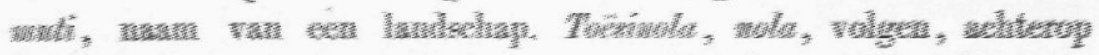
gaan. Leat, voetstappen. Fohan, sporen. Mai, 等onen. Nodr, brengen. Kedan, slechts. Daun, naald. Ida, een. Bua, pinangnoot. Humur, handvol. Tuli, waarde die men overeengekomen is. Lari, vijand. Aka, bladen. Dirun, tuaboom, Borassus flabelliformis. Neon, het hart, het inwendige. Kaikona, aangenaam, naar iemands smaak. Lalo-lalokan, overstelpt met allerlei gedachten. Kulan, zakdoek, overdrachtelijk ook maagden. Sai, uitgaan. Lesu, zakdoek; overdrachtelijk het gevolg bijv. van den dato, vorst. Beli, zingen. Odamatan, ingang van de steenen muren om de woningen der hoofden. Momoon, goed, behoorlijk. Keti, niet. Sidi, overtreden. Kiak, ach. Nuu, gelijk, Vatu, steen. Soëlerek, lerek, wegwerpen. Voti, opnemen, oprapen. Kokorek, kraaijen. Nalu, doen, maken. Koi, het gemoed, inwendige van den mensch. Tadu, onaangenaam, pijnlijk. Sadia, denken, er aan denken. Ina no aman, moeder en vader, ouders. 